\title{
Mantle and crust anisotropy in the eastern China region inferred from waveform splitting of SKS and PpSms
}

\author{
Takashi Iidaka ${ }^{1}$ and Fenglin Niu ${ }^{2}$ \\ ${ }^{1}$ ERI, University of Tokyo, Yayoi 1-1-1, Bunkyo-ku, Tokyo 113-0032, Japan \\ ${ }^{2}$ DTM, Carnegie Institution of Washington, 5241 Broad Branch Rd. NW, Washington, D.C. 20015, U.S.A.
}

(Received July 13, 2000; Revised November 30, 2000; Accepted December 8, 2000)

\begin{abstract}
$S$-waves converted from $P$-wave at different boundaries inside the earth, i.e., the Moho discontinuity and CMB, are used to determine the distribution of anisotropy in different layers. A clear later phase at approximately $17 \mathrm{sec}$ after the direct $P$-wave, which is identified to be PpSms (a phase that is $P$-to- $S$ converted at the free surface and is reflected by the Moho discontinuity on the receiver side), is observed in the radial component of seismograms recorded by broadband stations in the east China region. Waveform splitting observed from the PpSms and SKS suggests that the crust beneath the eastmost part of China is almost isotropic, and the mantle is weakly anisotropic. Splitting analysis using converted waves is a promising technique for investigating the depth distribution of shear-wave splitting.
\end{abstract}

\section{Introduction}

Although it is well known that elastic anisotropy of the earth is closely related to mantle dynamics, our knowledge of the depth distribution of anisotropy inside the earth is not sufficient to allow an understanding of the relation between anisotropy and mantle dynamics. Elastic anisotropy is studied seismologically by using waveform splitting, which is often observed in shear-wave arrivals. Most of the recent works on mantle and crustal anisotropy use teleseismic waves such as $S K S$ (e.g., Silver and Chan, 1991). Due to its $P$-to- $S$ conversion at the core-mantle boundary (CMB) on the receiver side, the waveform of $S K S$ is polarized only by the anisotropy on the receiver side. Global investigations (e.g., Silver and Chan, 1991; Vinnik et al., 1992; Zheng and Gao, 1994; Silver, 1996; Savage, 1999) suggest that the mean value of $\tau$, which is the delay time between the fast and slow polarized waves, for $S K S$ is about $1 \mathrm{sec}$. The observed splitting of $S K S$ waveforms is due to the accumulated effect of anisotropy on the receiver-side path including crustal anisotropy, although the time delay is usually interpreted as being due to be upper mantle anisotropy.

In general, identification of the source region of splitting has been a serious problem for shear-wave splitting analyses. Thus, although great heterogeneity of anisotropy with depth has been suggested, this heterogeneity remains to be demonstrated conclusively. Efforts to constrain the anisotropic region using seismological methods have been made by many researchers (e.g., Herquel et al., 1995; Vinnik and Montagner, 1996). Vinnik and Montagner (1996) used weak $P$-to- $S$ converted waves from stacked seismograms to determine anisotropy in the mantle transition zone. Iidaka and Niu (1998) have suggested a method to constrain upper

Copy right (C) The Society of Geomagnetism and Earth, Planetary and Space Sciences (SGEPSS); The Seismological Society of Japan; The Volcanological Society of Japan; The Geodetic Society of Japan; The Japanese Society for Planetary Sciences. and lower mantle anisotropy by comparing waveform of $S K S$ and P660s. P660s travels as a $P$-wave in the lower-mantle and is converted to an $S$-wave at the ' $660-\mathrm{km}$ ' discontinuity and travels as an $S$-wave in the upper mantle and crust. P660s wave is a converted wave at the upper and lower mantle boundary. Such waves can sometimes be observed on individual seismograms if the signal to noise ratio ( $\mathrm{S} / \mathrm{N}$ ratio) is sufficiently high. P660s and $S K S$ waves travel along almost the same ray path as an $S$-wave in the receiver-side upper mantle, but different paths in the lower mantle as a $P$ and an $S$-wave, respectively. Therefore, the difference in the waveform splitting between $S K S$ and P660s can be attributed directly to the anisotropy in the lower mantle where only $S K S$ travels as an $S$-wave. The $\tau$ values of the upper and lower mantle beneath eastern Asia were estimated to be about 0.2 and $0.4 \mathrm{sec}$, respectively (Iidaka and Niu, 1998).

Crustal anisotropy has been studied by many researchers and is globally observed to have large lateral heterogeneities (e.g., Kaneshima, 1990). Kaneshima and Ando (1989) used earthquakes that occurred in the crust and in the subducting plate beneath Japan to determine seismic anisotropy of the crust. However, it is very difficult to evaluate the contribution of all the crust to shear-wave splitting. The anisotropy observed from crustal earthquakes is due only to the upper crust because most of the crustal earthquakes are located in the upper crust. The observed anisotropy estimated from earthquakes in the subducting plate is comprised of the contribution from the upper mantle anisotropy and that from the crust.

Several shear-wave splitting studies have used $S K S$ waves to determine anisotropy in China (e.g., Silver and Chan, 1991; Zheng and Gao, 1994; Noda, 1995). Zheng and Gao (1994) studied shear-wave splitting for the whole of China. For most of the stations the fast polarization directions were close to the directions predicted from intraplate block deformation. Crustal anisotropy for China was studied by Gao et 


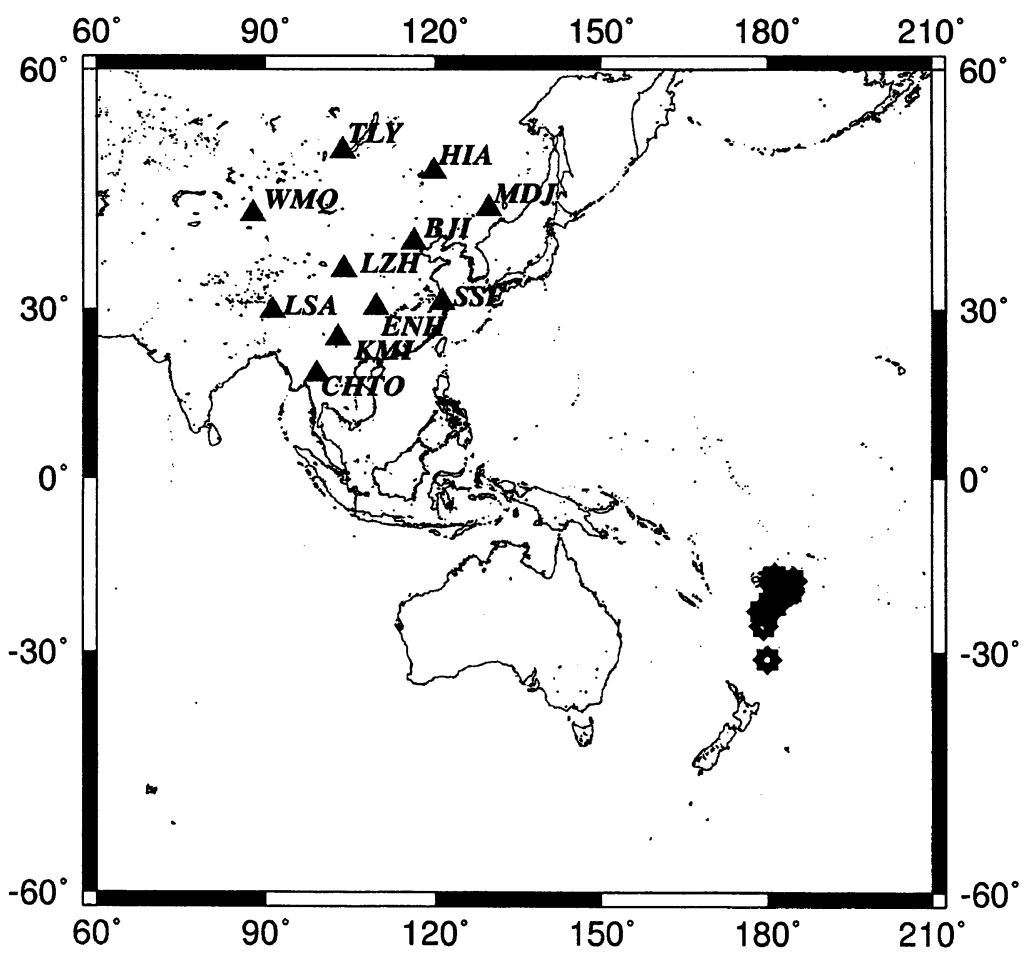

Fig. 1. Location map of the earthquakes (stars) and seismic stations (triangles).

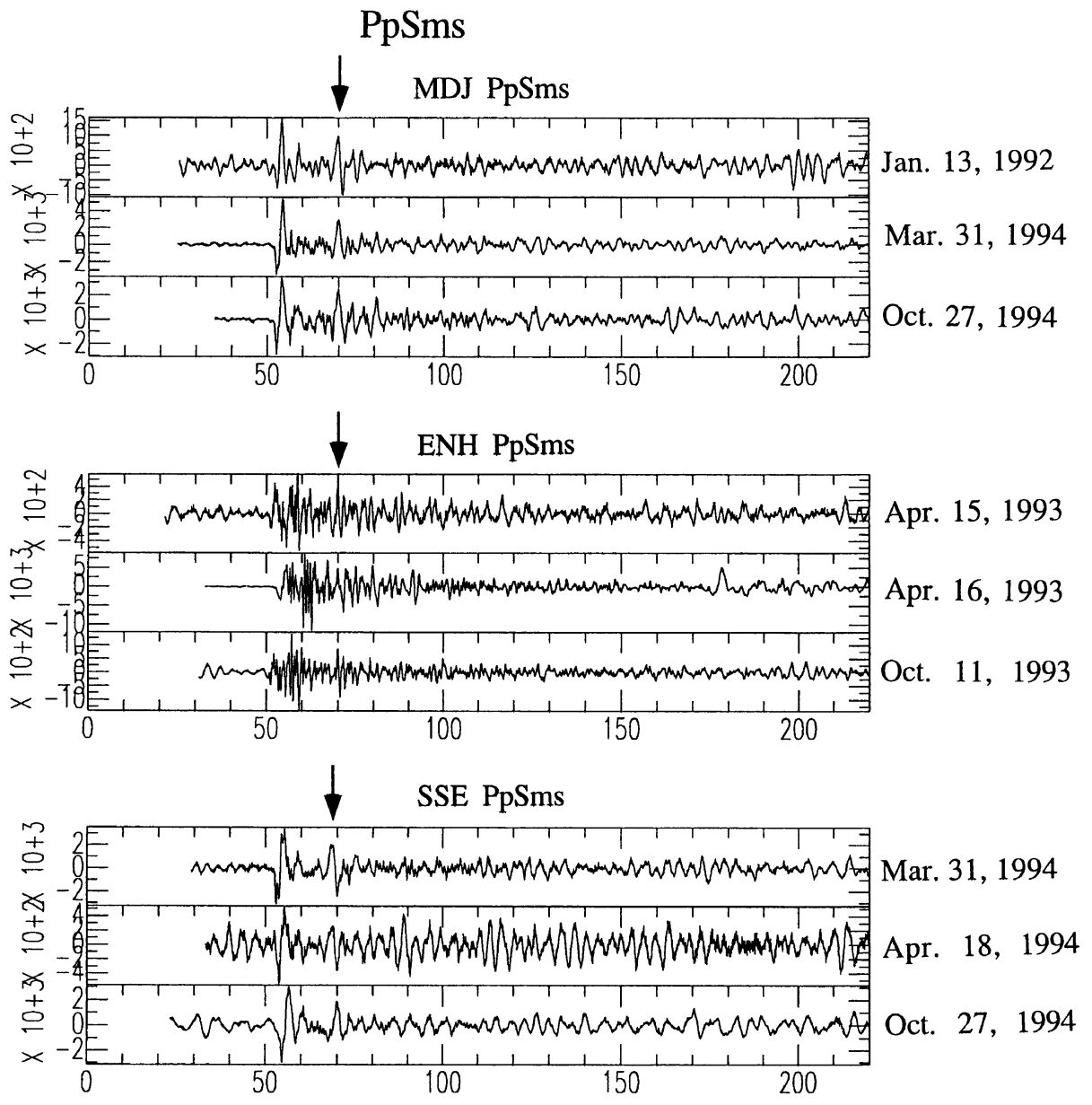

Fig. 2. Examples of the $X$-phase (arrows), which is identified as PpSms wave, at seismic stations MDJ, ENH, and SSE. 
Table 1. Earthquake list.

\begin{tabular}{|c|c|c|c|c|c|c|}
\hline $\begin{array}{c}\text { Date } \\
\text { (y m dy) }\end{array}$ & $\begin{array}{c}\text { Time } \\
\text { (h min s) }\end{array}$ & $\begin{array}{l}\text { Lat. } \\
\text { deg. }\end{array}$ & $\begin{array}{l}\text { Lon. } \\
\text { deg. }\end{array}$ & $\begin{array}{c}\text { Depth } \\
\text { km }\end{array}$ & $\begin{array}{l}\text { Mag. } \\
\text { Mw }\end{array}$ & \\
\hline 19900520 & 073236.9 & -18.09 & -175.34 & 232.0 & 6.3 & $\ddagger$ \\
\hline 19900528 & 112848.9 & -20.76 & -178.08 & 497.0 & 5.9 & $\ddagger$ \\
\hline 19900608 & 150510.2 & -18.70 & -178.90 & 499.0 & 6.5 & $*$ \\
\hline 19900828 & 031652.7 & -19.43 & -175.83 & 243.0 & 5.6 & $\ddagger$ \\
\hline 19901010 & 055458.0 & -23.35 & 178.87 & 594.0 & 6.1 & $\ddagger$ \\
\hline 19910609 & 074506.6 & -20.18 & -176.31 & 305.0 & 7.0 & $*$ \\
\hline 19910611 & 143248.0 & -18.13 & -178.43 & 628.0 & 5.6 & $\neq$ \\
\hline 19910613 & 171845.9 & -19.93 & -175.74 & 216.0 & 5.9 & $\ddagger$ \\
\hline 19910930 & 002147.5 & -20.97 & -178.61 & 580.0 & 7.0 & $*$ \\
\hline 19920113 & 093743.7 & -20.89 & -178.74 & 575.0 & 6.0 & $\sharp$ \\
\hline 19920711 & 104420.9 & -22.28 & -178.51 & 381.0 & 7.2 & $*$ \\
\hline 19930321 & 050459.0 & -17.97 & -178.53 & 584.0 & 6.3 & $\sharp$ \\
\hline 19930401 & 061116.0 & -19.87 & -177.55 & 406.0 & 5.7 & $\sharp$ \\
\hline 19930415 & 133238.4 & -18.08 & -178.12 & 458.0 & 5.6 & $\sharp$ \\
\hline 19930416 & 140838.8 & -17.69 & -178.88 & 568.0 & 6.9 & $* \sharp$ \\
\hline 19930420 & 162619.9 & -20.76 & -178.72 & 592.0 & 5.8 & $\sharp$ \\
\hline 19930512 & 115914.6 & -20.41 & -177.90 & 533.0 & 5.5 & $\sharp$ \\
\hline 19930709 & 153755.1 & -19.79 & -177.54 & 412.0 & 6.1 & $\sharp$ \\
\hline 19930807 & 175327.0 & -23.87 & 179.82 & 555.0 & 6.7 & $* \sharp$ \\
\hline 19931011 & 130729.9 & -17.80 & -178.76 & 556.0 & 6.0 & $\sharp$ \\
\hline 19940119 & 162648.4 & -17.50 & -178.59 & 537.0 & 6.1 & $\sharp$ \\
\hline 19940309 & 232807.7 & -17.77 & -178.50 & 564.0 & 7.6 & $* \sharp$ \\
\hline 19940331 & 224053.4 & -21.95 & -179.58 & 591.0 & 6.5 & $* \sharp$ \\
\hline 19940418 & 213943.1 & -21.37 & -178.83 & 541.0 & 5.9 & $\sharp$ \\
\hline 19940424 & 024131.0 & -20.95 & -178.79 & 584.0 & 5.7 & $\sharp$ \\
\hline 19941027 & 222031.0 & -25.79 & 179.35 & 549.0 & 6.7 & $* \sharp$ \\
\hline 19960805 & 223822.0 & -20.69 & -178.31 & 550.0 & 7.4 & $*$ \\
\hline 19961105 & 094134.7 & -31.16 & 180.00 & 369.0 & 6.8 & $*$ \\
\hline
\end{tabular}

$*$ : $S K S$ splitting parameters by this study.

$\sharp$ : PpSms splitting parameters by this study.

‡: SKS splitting parameters by Iidaka and Niu (1998).

al. (1995) using direct $S$-waves of shallow earthquakes. The crustal anisotropy was considered to be due to cracks.

Here, we extend our earlier study (Iidaka and Niu, 1998) to determine the anisotropy of mantle and crust by using another converted phase, PpSms (the notation is defined by Bath and Stefansson, 1966). PpSms is a later arriving $S$-phase that is converted from $P$ at the earth's surface, and reflected at the Moho discontinuity before arriving at a seismic station. PpSms makes two-way traverses through the crust along a near vertical path as an $S$-wave, and therefore samples the anisotropy in the crust twice. Because it does not travel in the mantle as a shear-wave, PpSms is free from the effects of mantle anisotropy.

In the eastmost China region, clear later arrivals ( $X$-phase) are observed at about $17 \mathrm{sec}$ after the first arrivals at seismic stations, ENH, MDJ, and SSE. The $X$-phase is identified as the PpSms wave, which is used to estimate the crustal anisotropy. The total anisotropy of the mantle and the crust is obtained from the waveform of SKS. Thus mantle anisotropy can be obtained by comparing the results for $S K S$ and PpSms.

\section{Data}

We use broadband data from IRIS Data Management Center for stations shown in Fig. 1. The coverage period is from January 1990 to December 1996 . The sampling rate of the data is $20 \mathrm{~Hz}$. A clear later arrival ( $X$-phase) appears about 17 sec after the direct $P$ arrivals on the seismograms at seismic stations MDJ, ENH, and SSE, eastern China (Fig. 2). The cause of the $X$-phase will be discussed and finally identified as PpSms in the following text. Earthquakes with impulsive PpSms arrivals are selected to avoid the artificial results caused by noise (Table 1).

$S K S$ is most easily observed beyond $80^{\circ}$. To avoid the contamination by other phases, such as $S, s S$, and $S c S$, we 
$03 / 31 / 94$

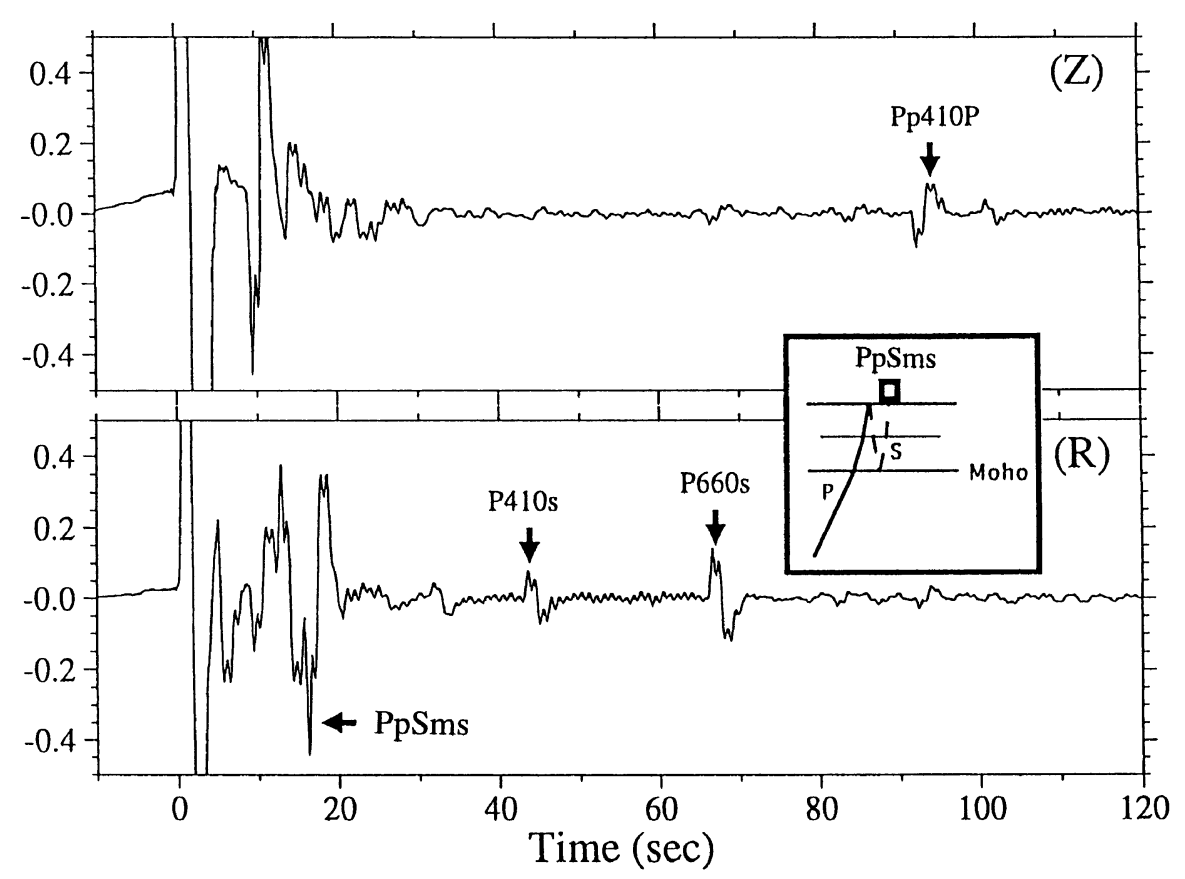

Fig. 3. Theoretical vertical $(Z)$ and radial $(R)$ component seismograms calculated for the iasp91 model when a $P$ plane wave arrives at a station from below with an incident angle of $35^{\circ}$ at a depth of $660 \mathrm{~km}$. Schematic ray path of $P p S m s$ wave is shown in the inset.

use earthquakes located deeper than $300 \mathrm{~km}$ with epicentral distance of $83^{\circ}-130^{\circ}$. Earthquakes with magnitudes larger than 6.5 are used (Table 1). The results of $S K S$ splitting data at HIA obtained by Iidaka and Niu (1998) are also added.

\section{Analysis}

\subsection{Identification of $X$-phase}

On seismograms of teleseismic earthquakes recorded by stations in the eastern China region, a clear later phase $(X$ phase) appears about $17 \mathrm{sec}$ after the first arrival (Fig. 2). The $X$-phase has the following characteristics. (1) Its amplitudes are largest on the radial component. (2) The $X-P$ times are not sensitive to the epicentral distance. (3) The $X$ - $P$ times are also not sensitive to the depth and location of the earthquakes. Possible interpretations are examined using a theoretical calculation, considering the converted and reflected waves at the known boundaries in the earth. Characteristic (3) indicates that the cause of the $X$-phase is not located in the source region. Characteristics (2) and (3) suggest that the cause of the $X$-phase is located near the station. Figure 3 shows theoretical $P$ and $S V$ seismograms calculated with the iasp91 model (Kennett and Engdahl, 1991) when a $P$ plane wave arrives at a station from below with an incident angle of $35^{\circ}$ at a depth of $660 \mathrm{~km}$, which corresponds to the approximate incident angle of $P$-waves arriving from Tonga at the stations of interest. The Thomson-Haskell method (Haskell, 1962) is used to construct synthetics. We notice that about 17 sec after the direct $P$-wave a clear crustal reverberation phase with large amplitude is shown on the $S V$-seismogram (Fig. 3, (R)). The theoretical calculation of the waveforms indicate the $X$-phase is well explained as a PpSms wave, which is a $P$-to- $S$ converted phase at the free surface that dives again into the earth and is finally reflected at the Moho discontinuity (Fig. 3, inset). The interpretation is also consistent with characteristic (1). We conclude the later phase is a PpSms wave. Shear-wave anisotropy for the whole crust is easily detected by analysis of PpSms waves.

\subsection{Shear-wave splitting detection}

Shear-wave splitting is usually expressed by two parameters which are time lag $\tau$ (in seconds), the time between the fast and slow components of the split shear wave, and the fast polarization azimuth $\phi$ (in degrees). We investigate shear-wave splitting using the techniques employed by Fukao (1984). After the seismic signals of SKS and PpSms waves are isolated from individual seismograms, we calculated the correlation for the two horizontal seismogram components over a grid $0^{\circ}$ to $180^{\circ}$ for $\phi$ and $0-8 \mathrm{~s}$ for $\tau$ with increments of $1^{\circ}$ and $0.05 \mathrm{~s}$, respectively. The time-lag $\tau$ (in seconds) and the fast polarization azimuth $\phi$ (in degree) are defined to be the values which yielded the maximum correlation (Fig. 4). The obtained values of azimuth and time lag are checked as follows. Anisotropy-corrected seismograms are obtained by a clockwise rotation $\phi$ and a relative time shift $\tau$. Appropriate rotation and time shift would show a linear orbit, which is expected to be in the radial direction, in the resultant particle motion diagram. The data indicating linear orbits obtained from anisotropy-corrected seismograms are used to exclude artificial results in which waveforms were distorted by noise. The average and standard deviation values of the observed shear-wave splitting at each seismic station are shown (Table 2).

\section{Results and Discussion}

One important advance in retrieving splitting information 
(A)



(b)

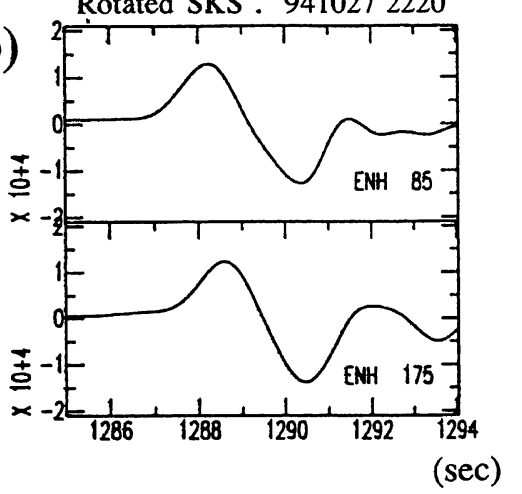

(B)

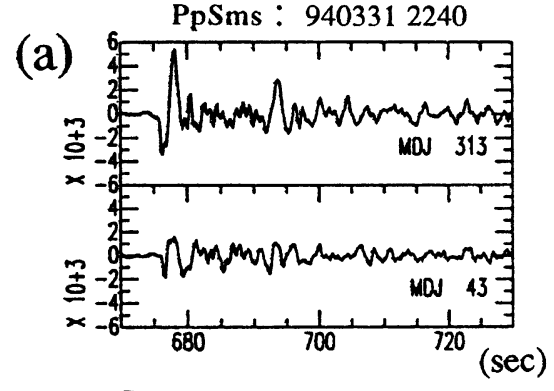

(b)

Rotated PpSms : 9403312240

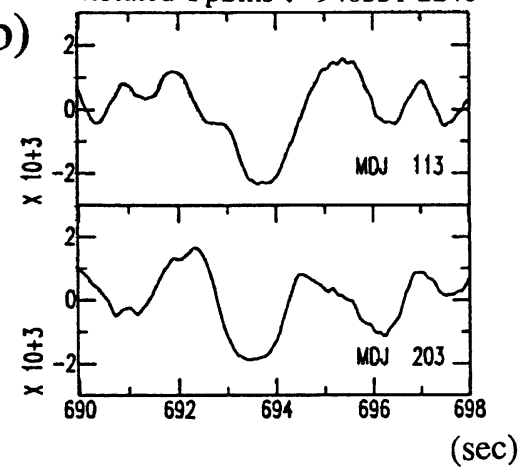

(c)

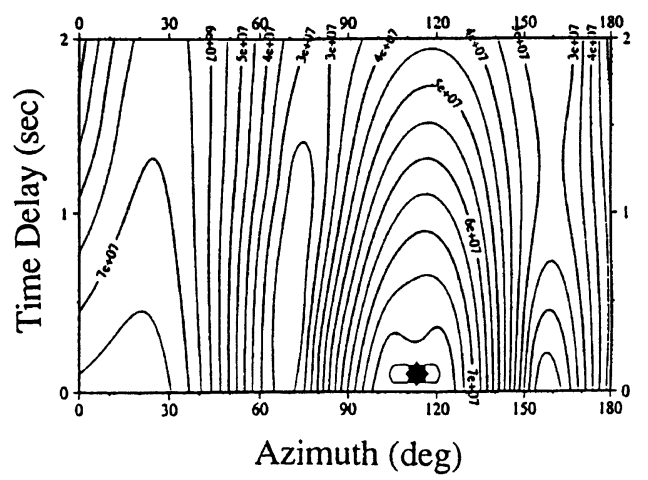

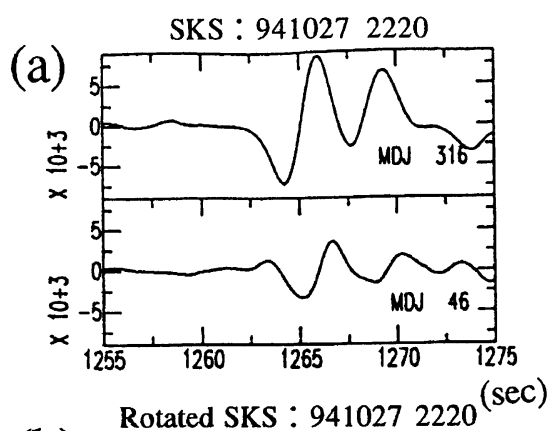



$(\mathrm{sec})$


(c)



Fig. 4. The rotated waveforms with the radial-transverse components (a) and maximum correlation (b) of the $S K S$ (A) and $P p S m s$ (B) waves. The contour maps of the cross-correlation function of PpSms are also shown (c). The maximum cross-correlation peak is shown by star. 




Fig. 5. Results of shear-wave splitting of $S K S$ at the eastern China region. Polarized azimuths of maximum-velocity phase and time delay between maximum and minimum velocity phases are shown by the direction and length of the bar, respectively. The absolute plate velocity directions of AM1-2 and HS2-NUVEL1 are shown by open and solid arrows, respectively (Kubo and Hiramatsu, 1998).

Table 2. Splitting parameters of $S K S$ and PpSms waves.

\begin{tabular}{lrrrcc}
\hline $\begin{array}{l}\text { Station } \\
\text { name }\end{array}$ & \multicolumn{1}{c}{$\begin{array}{c}\phi_{\text {Ave. }} \\
\text { deg. }\end{array}$} & $\begin{array}{c}\phi_{\text {S.D. }} \\
+/-\end{array}$ & $\begin{array}{c}\tau_{\text {Ave. }} \\
\text { sec. }\end{array}$ & $\begin{array}{c}\tau_{\text {S.D. }} \\
+/-\end{array}$ & $\begin{array}{c}\text { Num. of } \\
\text { data }\end{array}$ \\
\hline SKS & & & & & \\
BJI & 73.33 & 3.08 & 0.29 & 0.17 & 9 \\
CHTO & 62.57 & 5.62 & 0.27 & 0.16 & 7 \\
ENH & 75.57 & 7.36 & 0.36 & 0.13 & 7 \\
HIA & 2.60 & 7.50 & 0.56 & 0.10 & 10 \\
KMI & 70.00 & 9.79 & 0.29 & 0.17 & 9 \\
LSA & 69.63 & 7.58 & 0.19 & 0.09 & 8 \\
LZH & 135.83 & 45.22 & 0.14 & 0.11 & 7 \\
MDJ & 95.00 & - & 0.50 & - & 1 \\
TLY & 158.83 & 4.45 & 0.15 & 0.15 & 7 \\
WMQ & 71.78 & 5.29 & 0.46 & 0.19 & 9 \\
$\boldsymbol{P p S m s}$ & & & & & \\
ENH & 55.29 & 10.89 & 0.03 & 0.02 & 9 \\
MDJ & 13.20 & 13.66 & 0.03 & 0.03 & 8 \\
SSE & 89.00 & 21.63 & 0.05 & 0.04 & 4 \\
\hline
\end{tabular}

S.D.: Standard Deviation.

—: No standard deviation data because of only one result.

has been the utilization of $S K S$. This phase was exploited extensively in teleseismic splitting (e.g., Silver and Chan, 1991; Vinnik et al., 1992), because it has many advantages for studies of the continents. Stable values of $\phi$ and $\tau$ are obtained from the analysis of $S K S$ waves (Fig. 5, Table 2). We divide these anisotropic data into two groups: one has a fast polarization in the north-south direction observed at the northern part of the studied area (TLY, and HIA), the other

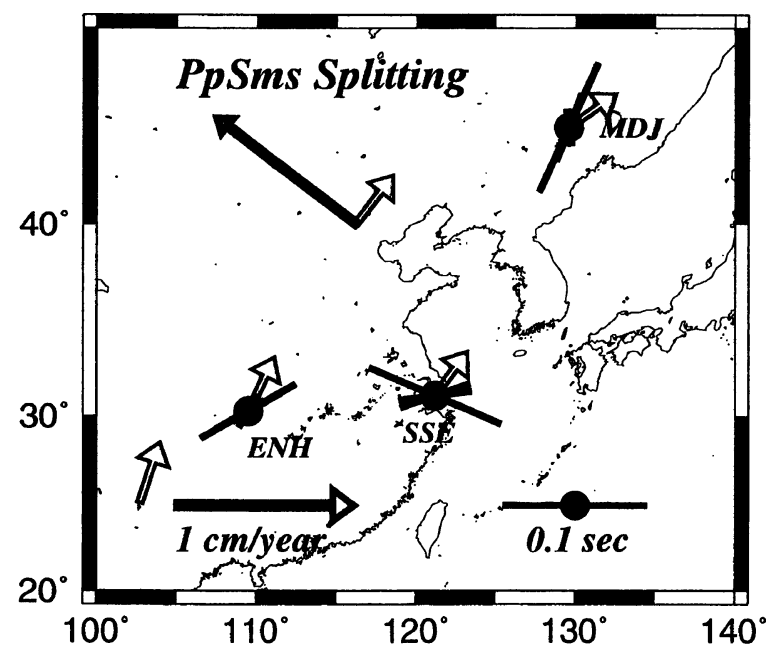

Fig. 6. Results of shear-wave splitting of PpSms at eastmost China. Polarized azimuths of maximum-velocity phase and time delay are shown by the direction and length of the bar, respectively. The absolute plate velocity directions of AM1-2 (open arrows) and HS2-NUVEL1 (solid arrows) are shown (Kubo and Hiramatsu, 1998).

has a fast polarization in the ENE-WSW direction obtained for the southern part of the studied area (CHTO, KMI, LSA, ENH, BJI, MDJ, and WMQ). The ENE-WSW polarizations are stable over a large area with $\tau$ values of about $0.2 \mathrm{sec}-$ $0.5 \mathrm{sec}$.

The polarization direction and delay time results at station of HIA are generally close to those obtained previously (e.g., Zheng and Gao, 1994). But for some other stations, our observed values are different from previous results. Among the previous studies, Noda (1995) used only Tonga events, while others used many earthquakes located all over the world to cover the wide back-azimuth ranges. Our results are very 


\section{A)}



B)





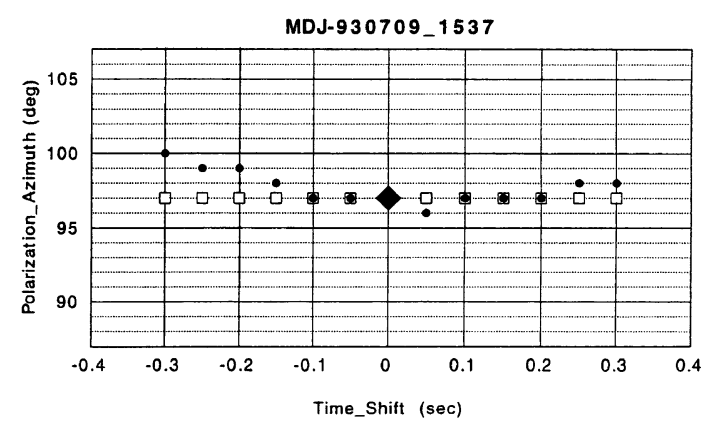

Fig. 7. Results of the simulation that re-estimated shear-wave splitting parameters (A: time-lag $\tau$, B: the fast polarization azimuth $\phi$ ) using time-shifted waveforms. The large diamonds denote original results of the shear-wave splitting. The open squares indicate time-shifted values. The solid circles suggest re-calculated shear-wave splitting using time-shifted waveforms.

close to the observed values of Noda (1995), and are different from those of the others. If anisotropic regions exist in the deeper part of the mantle (Iidaka and Niu, 1998), and they are heterogeneously distributed, then using a dataset with a good back-azimuthal coverage simply means averaging anisotropy from different $S K S$ ray paths. Our data are from earthquakes located only in Tonga region. The splitting results from Tonga earthquakes could be different from those of averaged values.

The observed splitting parameters of $S K S$ waves result from the accumulated effects of anisotropy in the lower mantle, upper mantle and crust. It is difficult to isolate contributions from different parts of the earth (crust, upper mantle, and lower mantle) from the cumulative splitting values. The shear-wave splitting of PpSms is a manifestation of crustal anisotropy. PpSms phases were observed at stations ENH, $\mathrm{SSE}$, and MDJ, located along the east coast of China. The results of shear-wave splitting of PpSms are shown (Fig. 6, Table 2). The fast polarization directions of ENH and MDJ are ENE-WSW and NNE-SSW, respectively, and the fast polarization direction observed at SSE is almost east-west. The averaged $\tau$ values of the shear-wave splitting at these stations are less than $0.05 \mathrm{sec}$, which indicates that the anisotropy caused by the crust is less than $0.05 \mathrm{sec}$. Gao et al. (1995) investigated the splitting of direct $S$-waves from crust earthquakes with focal depth of about $10 \mathrm{~km}$ in Tangshan region, north China. Their obtained delay times are in the range of $0.001 \mathrm{sec} / \mathrm{km}-0.0258 \mathrm{sec} / \mathrm{km}$. Thus, the delay time for a 10 $\mathrm{km}$ thick layer in the upper crust is in the range of $0.01 \mathrm{sec}-$ $0.258 \mathrm{sec}$. Our observed crustal anisotropy with small $\tau$ values is consistent with the previous results obtained in China. This is also consistent with the results of Kaneshima (1990), who reported a $\tau$ value of crustal anisotropy beneath Japan in the range of $0 \mathrm{sec}-0.2 \mathrm{sec}$. Comparing our results with the results of Gao et al. (1995), we might infer that lower crust beneath the eastmost China region is weakly anisotropic or almost isotropic. A similar result was reported in Japan and was used to conclude that the source of crustal anisotropy is located in the upper crust (Kaneshima and Ando, 1989).

The maximum $\tau_{0}$ value of PpSms ( $\tau=\tau_{0} / 2 ; \tau_{0}$, the original time difference between the fast and slow polarized waves of PpSms, reflects twice the crustal anisotropy because the PpSms makes a two-way traverse through the crust) is 0.2 sec. The maximum time difference of $0.2 \mathrm{sec}$ between the fast and slow polarized waves is equivalent to 4 samples on the digital data because the $20 \mathrm{~Hz}$ sampling data are used. The averaged time lag $\tau$ values are small and scattered. However, PpSms waves on the radial components are large amplitude with high $\mathrm{S} / \mathrm{N}$ ratio on the seismograms. The maximum cross-correlated waveforms are very similar and the particle motion is almost purely in the radial direction. The crosscorrelation function also suggests a clear peak (Fig. 4(c)). Thus our suggestion of an isotropic crust in this region is supported by the data.

We also evaluate the accuracy of the small shear-wave splitting value using the following simulation. One of the two 


\section{A)}



\section{B)}

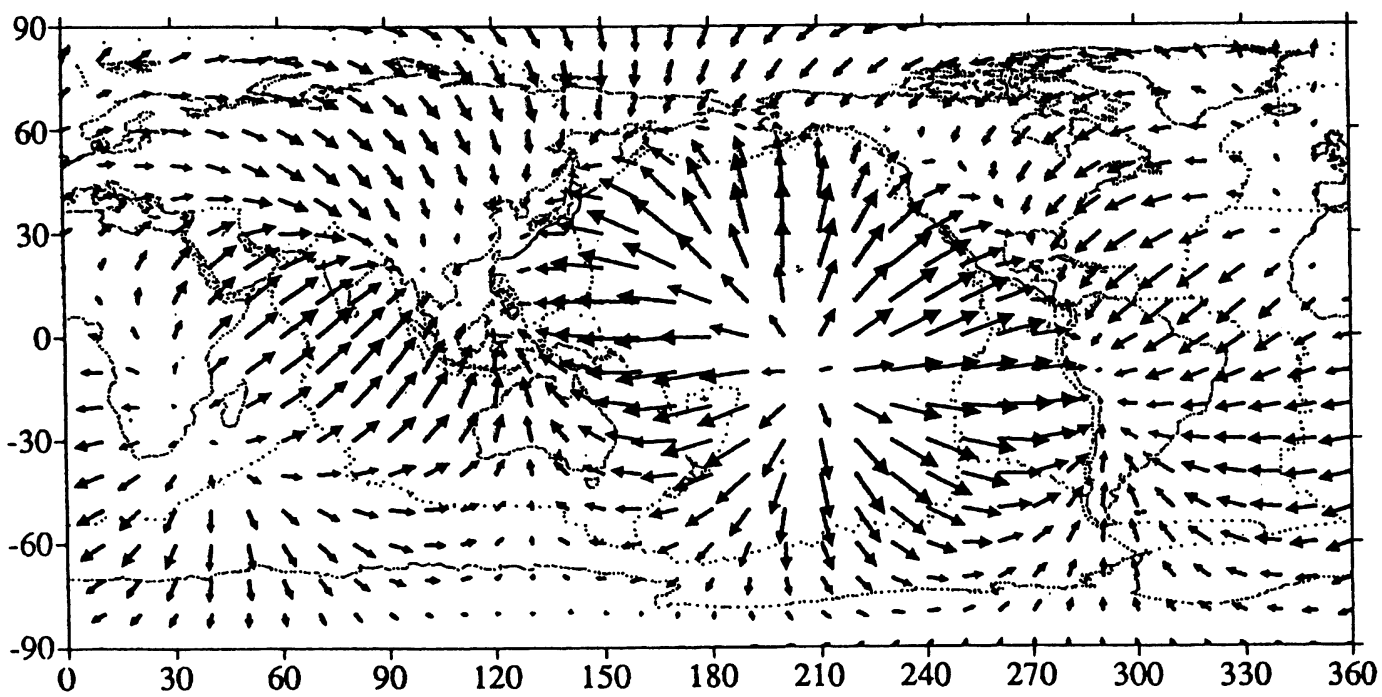

Fig. 8. A) Map showing of the distribution of main active faults in China (Wang et al., 1998). B) Predicted convective flow pattern based on the seismic tomography data (Kido, 1994).

components with maximum cross-correlation is successively shifted by one sampling interval from $-0.3 \mathrm{sec}-+0.3 \mathrm{sec}$ on the time axis. The shifted-waveform and its orthogonalcomponent waveform are rotated to the north-south and eastwest component. The same process, which was used to determine the splitting parameters for original observed data, is also applied to detect shear-wave splitting on the time-shifted waves. If the resolution in time axis is sufficient to detect the small $\tau$ value, which is equivalent to several sampling intervals, our shear-wave splitting analysis should detect the shifted-time values. The results of the simulation are shown in Fig. 7. The $\tau$ values obtained from original waveforms are shown by large diamonds. The theoretical time-shift values are shown by small open squares. Reproduced splitting times using the time-shifted waves are shown by solid circles. If the accuracy is sufficient to detect $\tau$ value variations 
equal to one data sampling interval, the open squares and solid circles should overlap. The results of the simulation show that the obtained $\tau$ values are stable with small errors of less than $0.05 \mathrm{sec}$ and the polarization azimuth is also recovered with a scatter of less than 5 degrees in the case of the shifted-time data. Therefore, the simulation suggests that the obtained $\tau$ values, which are equivalent to several data sampling intervals, are convincing. The resolution of our analysis is adequate for detection of anisotropy with a time split magnitude of several samples.

There are three possible explanations for the observed small $\tau$ values: 1) the crust in the studied region is isotropic; 2) crustal anisotropy with a vertical axis of symmetry (transverse isotropy); 3) the radial directions from Tonga earthquakes to the eastern China stations happen to be along the fast or slow polarization direction of the anisotropic crust beneath the stations. The cases of 1) and 2) indicate the region is horizontally isotropic. As for the third case, since the radial directions are neither parallel nor at right angles to the apparent fast polarization directions, we conclude that the observed very small values of $\tau$ from PpSms waves are reliable and the crustal anisotropy in the studied region is very small.

Causes of shear-wave splitting have been proposed by many scientists. Silver and Chan (1991) suggested that mantle anisotropy was dominated by the latest significant episode of internal coherent deformation of the continental lithosphere by tectonic processes such as orogenies, rifting episodes, and strike-slip deformation. On the other hand, Vinnik et al. (1992) suggested that the polarization directions in tectonic areas and stable continents reflect the local tectonic structure and the directions of plate motion, respectively. Meanwhile, Savage (1999) suggested that both lithospheric and asthenospheric deformations might be the cause of observed shear-wave splitting.

The spatial pattern of the main active faults in China is very complex (Wang et al., 1998) (Fig. 8(A)). The polarization directions at ENH and MDJ are roughly consistent with the direction of the main active faults. However, the observed splitting data cannot be completely explained by the trends of main active faults. Moreover, our results suggest the crustal anisotropy contribution to the observed shear-wave splitting of $S K S$ is small, so that the source region of the anisotropy of $S K S$ is located mainly in the mantle.

The close relationship between shear-wave polarizations and the preferred orientation of anisotropic crystals provides a method for studying physical properties of the upper mantle (e.g., Crampin, 1981). Mantle anisotropy observed in most regions can be explained by an alignment of olivine due to mantle flow. Kubo and Hiramatsu (1998) calculated the absolute plate velocity direction at seismic stations in China based on the AM1-2 model (Minster and Jordan, 1978) and the HS2-NUVEL1 model (Gripp and Gordon, 1990) (Figs. 5 and 6). The fast polarization directions at the seismic stations in the eastern China are not consistent with the absolute plate velocity directions.

The present mantle convective flow can be calculated from results of tomographic studies (e.g., Vigny et al., 1991; Kido, 1994). The seismic tomographic studies have mapped lateral variations in seismic velocities with a resolving power of several thousands kilometers. The three-dimensional pattern of the mantle which depicts the present distribution of buoyancy is translated into density variations. The heterogeneous density variations cause mantle convective flow. The predicted flow estimated by Kido (1994) is shown (Fig. 8(B)). The present convective flow at the northern part of China was estimated to be in the south direction with lateral variations. For eastern China, a small flow with a southwest direction was obtained. The pattern of polarization directions from $S K S$-wave analysis is similar to those of the predicted flows. The observed shear-wave splitting therefore might be related to convective flow caused by density heterogeneity.

From our results, the crust is almost isotropic, and whole the mantle anisotropy is estimated to be about $0.2 \mathrm{sec}-0.5$ sec. When evaluating the magnitude of anisotropy at each layer by using different converted waves, one must allow for the frequency dependent effects on the observed anisotropic parameters, as well as possible effects caused by multiple anisotropic layers and heterogeneity (e.g., Marson-Pidgeon and Savage, 1997). The converted phases could also be modified by dipping boundaries in flat-layered anisotropic medium (Levin and Park, 1997; Savage, 1999), which might affect the determination of splitting parameters. However, our observation of PpSms is very robust, and we do not expect that our measurement could be significantly affected by the above elements.

\section{Conclusions}

By comparing waveform splitting of $S K S$ and PpSms, we are able to estimate the possible contributions from different layers to the observed anisotropy. Analysis of PpSms waves, observed at seismic stations ENH, MDJ, and SSE, indicates that the crust beneath the eastmost part of the China region is almost isotropic. The observed shear-wave splitting of $S K S$ is caused mainly by an anisotropic region located in the mantle. Delay times through the whole mantle beneath the stations are estimated to be $0.2 \mathrm{sec}-0.5 \mathrm{sec}$. Splitting analysis using converted waves is a promising technique for investigating the depth distribution of shear-wave splitting.

Acknowledgments. We thank Dr. Kaneshima and Prof. Kawakatsu for helpful discussions. SAC (Seismic Analysis Code) was used in our calculation. We also thank Drs. Kubo and Hiramatsu for providing plate motion data. This research was supported by a grant from "Ocean Hemisphere Network Project" by Japanese Ministry of Education, Science and Culture. We thank Dr. Linde for critically reading the manuscript, and Dr. Savage and an anonymous reviewer for constructive reviews.

\section{References}

Bath, M. and R. Stefansson, S-P conversion at the base of the crust, Annali di Geofisica, 19, 119-130, 1966.

Crampin, S., A review of wave motion in anisotropic and cracked elasticmedium, Wave Motion, 3, 343-391, 1981.

Fukao, Y., Evidence from core-reflected shear waves anisotropy in the Earth's mantle, Nature, 309, 695-698, 1984.

Gao, Y., S. Zheng, and Y. Sun, Crack-induced anisotropy in the crust from shear wave splitting observed in Tangshan region, North China, Acta Seism. Sinica., 8, 351-363, 1995.

Gripp, A. E. and R. G. Gordon, Current plate velocities relative to the hotspots incorporating the NUVEL-1 global plate motion model, Geophys. Res. Lett., 17, 1109-1112, 1990.

Haskell, N. A., Crustal reflections of the plane $P$ and $S V$ waves, J. Geophys. Res., 67, 4751-4767, 1962. 
Herquel, G., G. Wittlinger, and J. Guilbert, Anisotropy and crustal thickness of Northern-Tibet, New constraints for tectonic modeling, Geophys. Res. Lett., 22, 1925-1928, 1995.

Iidaka, T. and F. Niu, Evidence for an anisotropic lower mantle beneath eastern Asia: Comparison of shear-wave splitting data of $S K S$ and P660s, Geophys. Res. Lett., 25, 675-678, 1998.

Kaneshima, S., Original of crustal anisotropy: Shear wave splitting studies in Japan, J. Geophys. Res., 95, 11121-11133, 1990.

Kaneshima, S. and M. Ando, An analysis of split shear waves observed above crustal and uppermost mantle earthquakes beneath Shikoku, Japan, Implications in effective depth extent of seismic anisotropy, J. Geophys. Res., 94, 14077-14092, 1989.

Kennett, B. L. N. and E. R. Engdahl, Travel times for global earthquake location and phase identification, Geophys. J. Int., 105, 429-465, 1991.

Kido, M., On the present convective pattern in the mantle predicted by seismic tomography, Zisin , 47, 411-421, 1994 (in Japanese with English abstract).

Kubo, A. and Y. Hiramatsu, On presence of seismic anisotropy in the asthenosphere beneath continents and its dependence on plate velocity Significance of reference frame selection, Pure Appl. Geophys., 151, 281303, 1998.

Levin, V. and J. Park, P-SH conversions in a flat-layered medium with anisotropy of arbitrary orientation, Geophys. J. Int., 131, 253-266, 1997.

Marson-Pidgeon, K. and M. Savage, Frequency-dependent anisotropy in Wellington, New Zealand, Geophys. Res. Lett., 24, 3297-3300, 1997.

Minster, J. B. and T. H. Jordan, Present-day plate motions, J. Geophys. Res.,
83, 5331-5354, 1978 .

Noda, T., SKS anisotropy using data of IRIS stations, Masters thesis, Kobe Univ., 52 pp., Kobe, Japan, 1995 (in Japanese).

Savage, M. K., Seismic anisotropy and mantle deformation: What have we learned from shear wave splitting?, Rev. of Geophys., 27, 65-106, 1999.

Silver, P. G., Seismic anisotropy beneath the continents: probing the depths of Geology, Ann. Rev. Earth Planet. Sci., 24, 385-432, 1996.

Silver, P. G. and W. Chan, Shear wave splitting and subcontinental mantle deformation, J. Geophys. Res., 96, 16429-16454, 1991.

Vigny, C., Y. Richard, and C. Froidevaux, The driving mechanism of plate tectonics, Tectonophys., 187, 345-360, 1991.

Vinnik, L. P. and J. P. Montagner, Shear wave splitting in the mantle Ps phases, Geophys. Res. Lett., 18, 2449-2452, 1996.

Vinnik, L. P., L. I. Makeyeva, A. Milev, and Y. Usenko, Global patterns of azimuthal anisotropy and deformation in the continental mantle, Geophys. J. Int., 111, 433-447, 1992.

Wang, J., X. Xhu, and Y. Xu, Fractal analysis applied to faults and earthquakes-A case study of China-, Acta Seism. Sinica., 11, 349-353, 1998.

Zheng, S. and Y. Gao, Azimuthal anisotropy in lithosphere on the Chinese mainland from observations of SKS at CDSN, Acta Seism. Sinica., 7, 177-186, 1994

T. Iidaka (e-mail: iidaka@eri.u-tokyo.ac.jp) and F. Niu (e-mail: niu@, dtm.ciw.edu) 